\title{
Neurological connections and endogenous biochemistry - potentially useful in electronic-nose diagnostics for coronavirus diseases
} \author{
Matthew Palm ${ }^{4}$ \\ 'Department of Electrical Engineering, University of South Florida, Tampa, FL 33620, USA \\ ${ }^{2}$ Department of Medical Engineering, University of South Florida, Tampa, FL 33620, USA. \\ ${ }^{3}$ Muma College of Business, University of South Florida, Tampa, FL 33620, USA. \\ ${ }^{4}$ Valhall K-9 International, LLC, Hull, GA 30646, USA.
}

Tiffany C. Miller ${ }^{1}$, Salvatore D. Morgera ${ }^{1}$, Stephen E. Saddow ${ }^{1,2}$, Arash Takshi $^{1}$, Matthew Mullarkey ${ }^{3}$,

Correspondence to: Dr. Salvatore D. Morgera, Department of Electrical Engineering, University of South Florida, 4202 E. Fowler Ave., Tampa, FL 33620, USA. E-mail: sdmorgera@usf.edu

\begin{abstract}
How to cite this article: Miller TC, Morgera SD, Saddow SE, Takshi A, Mullarkey M, Palm M. Neurological connections and endogenous biochemistry - potentially useful in electronic-nose diagnostics for coronavirus diseases. Neuroimmunol Neuroinflammation 2021;8:284-96. https://dx.doi.org/10.20517/2347-8659.2021.05
\end{abstract}

Received: 23 Feb 2021 First Decision: 24 May 2021 Revised: 11 Jun 2021 Accepted: 29 Jun 2021 First online: 21 Dec 2021

Academic Editors: Jin-Bin Xu, Athanassios P. Kyritsis Copy Editor: Xi-Jun Chen Production Editor: Xi-Jun Chen

\begin{abstract}
As our understanding of infectious diseases, such as coronavirus diseases including, Coronavirus Disease 2019 (COVID-19), as well as human respiratory viral and nonviral diseases, improves, we expect to uncover a better understanding of the pathogenesis of the disease as it relates to neuroinflammation. This may include associated biomarkers of immune response for neuroinflammation, central nervous system injury, and/or peripheral nervous system injury emitted from the breath and/or odor of an individual. Electronic nose gas sensing technology may have the potential to substantially slow the spread of contagious diseases with rapid diagnostic signal indication for detecting these emitted biomarkers of disease. The biochemistry behind the disease is critical for revealing the target gasses emitted in the breath of a severe acute respiratory syndrome coronavirus (SARS-CoV-2) infected individual for the development of such a selective diagnostic screening tool. In this paper, we comprehensively review the evidence that SARS-CoV-2 infection involves an inflammatory response mechanism involving the olfactory nerve route in the brain from the nasal canal suggesting potential candidate volatile organic compound target gas biomarkers, forming the breath pattern signature of COVID-19, that may be detected by electronic nose technologies from a breath sample.
\end{abstract}


Keywords: COVID-19, biomarkers, neuroinflammation, volatile organic compounds, CNS disease, inflammatory response

\section{INTRODUCTION}

In response to problems in the field investigated in recent studies by breath specialists based in Edinburgh, UK, in Dortmund, Germany ${ }^{[1]}$, and in Jacksonville, Florida, United States, a weekly virtual multidisciplinary meeting was established at the University of South Florida in April 2020 in collaboration with canine detection specialists Valhall K-9 International, LLC to discuss and begin understanding neurological manifestations of coronavirus diseases including, Coronavirus Disease 2019 (COVID-19), and potential diagnostic breath sensor devices in an attempt to address a long felt, yet unfulfilled need to standardize electronic nose methodologies into a reproducible method for detection of low noise data signature profiles of disease ${ }^{[2]}$. In particular, brain breath biochemistry and its associated biomarkers of neuroinflammation will be the main focus of this paper as they show the most pertinent relevance to candidate endogenous compounds of a COVID-19 breath pattern signature.

This review paper will first describe symptoms and the standard diagnostic testing method of COVID-19. Next, the clinical detection of COVID-19 in a patient's breath by various electronic nose devices will be discussed. Third, brain breath biochemistry in neurological complications of COVID-19 will be discussed including the infection mechanism of severe acute respiratory syndrome coronavirus (SARS-CoV-2) with a specific focus on its angiotensin-converting enzyme 2 (ACE2) interaction, viral infection routes from the olfactory epithelium at the nasal cavity to the central nervous system (CNS). Fourth, neurological manifestations of COVID-19 will be detailed including, COVID-19 Induced Pediatric Multisystem Inflammatory Syndrome (PMIS). Finally, the conclusion and future work in the development of an electronic nose for detecting COVID-19 will be described in its potential to determine a specific disease from a complex mixture of volatile organic compound (VOC) inflammatory biomarkers.

\section{COVID-19 SYMPTOMS AND DIAGNOSTIC TESTING}

\section{Symptoms associated with COVID-19}

On March 11, 2020 SARS-CoV-2 infection has facilitated a respiratory disease pandemic known as COVID19. Some symptoms of COVID-19 that have been observed in infected individuals comprise anosmia and ageusia. Anosmia is characterized by a drastic reduction of function the olfactory system, whereby, the detection of odors is diminished or lost. It is known that the ageusia symptom features the inability to tast $^{[3,4]}$. Although a loss of smell has been associated with neurodegenerative diseases from other pathogens prior to the COVID-19 pandemic, both anosmia and ageusia have become an increasingly known observed symptom of infection from severe acute respiratory syndrome coronavirus (SARS-CoV) or SARS-CoV-2 which causes COVID-19. Thus, understanding the biomechanism of anosmia may provide anosmia specific biomarkers capable of being added to the known breath pattern signature of COVID-19 currently consisting of increases in acetone, isoprene, heptanal, propanol, propanal, butanone, octanal with a decrease on methanol, thereby, differentiating COVID-19 infection from other pathogens of disease ${ }^{[1]}$. The incorporation of additional biomarkers from the anosmia symptom of COVID-19 contributes to the unique exhaled breath profile of COVID-19 to increase the accuracy of a diagnostic electronic nose for exhaled breath applications. Although SARS-CoV-2 infection causes COVID-19, studies have indicated a plurality of viruses access the CNS and/or the peripheral nervous system by traversing the length of the olfactory nerve. Once the virus has been incorporated within the nervous system, the activation of T lymphocytes triggers an inflammatory response from microglia and other inflammatory mediators ${ }^{[5,6]}$. This inflammatory response generates biomarker byproducts that are released into the surrounding environment through 
many pathways. For example, the lungs may generate disease specific metabolites that are exhaled through a breath. This breath sample may be analyzed by an electronic nose for abnormal chemical biomarkers that are associated with a disease ${ }^{[7]}$.

\section{Diagnostic testing of COVID-19}

Due to the highly contagious and life-threatening characteristics of COVID-19, the Emergency Use Authorization authority has allowed the use of real-time reverse transcription polymerase chain reaction (rRT-PCR) testing to detect RNA from SARS-CoV-2 in nasal, nasopharyngeal, and oropharyngeal swabs from patients exhibiting symptoms of the virus. This rRT-PCR method requires target-specific fluorescent labeled oligonucleotide probes which create a signal when they are bound to the amplified $\mathrm{RNA}^{[8]}$. This lengthy procedure requires many steps during preparation of the sample including, but not limited to, collecting samples from nasal swabs, providing quinidine isothiocyanate to disrupt the SARS-CoV-2 virions, providing a magnetic nanoparticle to capture the nucleic acids, washing the sample to remove inhibitors and unbound sample components, providing a nucleic acid detection kit for separating the nucleic acids from the magnetic nanoparticle with a buffer solution, amplifying the nucleic acids in a deep well plate, an internal control reagent is introduced to the wells and the specimen is plated, amplified, and detected on a reaction plate ${ }^{[8]}$. This diagnostic process requires many materials associated with an increase in cost and is subject to human error along the process which may adversely affect the accuracy of the testing result. Further, between April-June 2020, there have been shortages of genetic extraction kits, swabs, reagents, masks, and gowns which have halted COVID-19 testing.

Many countries have been implementing reverse transcription-polymerase chain reaction PCR COVID-19 testing of its population to reduce the spread of COVID-19. Studies have revealed that lockdowns and mobility reductions decreased the transmission rate of COVID-19 in Europe and North America ${ }^{[9]}$. However, manufacturers of the current polymerase chain reaction (PCR) COVID-19 diagnostic test kits struggle to keep up with demand and experience testing kit shortages and long processing times ${ }^{[10]}$. As a result, test results may be delayed which then delays the intervention of quarantining the potentially infected individual who may be transmitting viral particles within the wait period for their test results, which is typically 3 or more days. Thus, there is a need for a rapid COVID-19 screening test that is capable of providing results in real-time so that a confirmed COVID-19 infected individual can be alerted of their infection status within seconds of taking the test. A confirmed COVID-19 positive individual would be immediately advised by a healthcare professional, at point of care, to quarantine and isolate to slow the spread of infection, so that businesses and schools can continue providing services in a COVID-19 free environment.

Although rRT-PCR is the current standard and diagnostic testing method due to its high sensitivity having an approximate range between 66\%-83\% for detecting the RNA of SARS-CoV-2, studies indicate falsenegative (FN) results from respiratory samples for SARS-CoV-2 and show FN rates (FNRs) having a range between $1 \%$ to $30 \%$ due to, for example, swab contamination from poor sample collection, low viral load, different rates of viral shedding over time in the disease process, and decreased analytic sensitivity ${ }^{[1,12]}$. Thus, the substantially increased range of FNRs with rRT-PCR test results serves as a motivation for a diagnostic electronic nose breath analysis testing method. In particular, it depending on the type of electronic chemical gas sensors used and the amount of these sensors that comprise a rapid electronic nose device, the high selectivity of these sensors to the breath pattern signature of coronavirus disease would greatly reduce the number of false positives and false negatives that have been demonstrated by existing rRT-PCR diagnostic testing methods. 
Further, it would be more desirable to have a method of COVID-19 testing that eliminates the need for excessive equipment, supplies, and multiple steps. Since there have been shortages of testing materials such as swabs and associated reagents to perform rRT-PCR, some non-electronic nose investigations have sought out COVID-19 patient breath samples for processing and COVID-19 VOC signature detection with a gas chromatograph ion mobility spectrometer device. Evidence suggests a signature of inflammatory and oxidative stress metabolites detected from COVID-19 positive patients using gas chromatography (GC) and ion mobility spectrometry (IMS) include, acetone, alcohol, butanone, methanol, isoprene, heptanal, propanol, propanal and octanal ${ }^{[1]}$. Other studies have indicated VOC concentrations of approximately 10 to 250 parts-per-billion of methylpent-2-enal, 2,4-octadiene, 1-chloroheptane, and nonanal in breath samples obtained from exhaled breath samples of COVID-19 infected patients ${ }^{[1,1,14]}$.

\section{ELECTRONIC NOSE DETECTION OF BIOMARKERS OF RESPIRATORY DISEASE}

Electronic noses have been shown to successfully recognize complex VOC mixtures from a sample breath indicative of a metabolic process in respiratory diseases. Many electronic nose devices comprise an electronic chemical sensor array configured for detection of a target gas, such as VOCs, and implements pattern recognition to differentiate the target gas from a diversity of gasses ${ }^{[15]}$. Current existing electronic nose technologies have been developed and investigated for their diagnostic performance in respiratory diseases including, but not limited to, inflammatory lung disease such as asthma ${ }^{[16,17]}$. For example, the Cyranose 320 is an electronic nose having a carbon black-polymer sensor array ${ }^{[17]}$. Studies have investigated the performance of the Cyranose 320, configured for 96\% accuracy in distinguishing patients with asthma from patients with chronic obstructive pulmonary disease ${ }^{[16]}$. Another study indicated the Cyranose 320 could differentiate patients having mild asthma from patients having severe asthma at a cross-validation value of $65 \%^{[17,18]}$. In yet another study, the Cyranose 320 was shown to discriminate between healthy controls, controlled asthma, and partially-controlled and uncontrolled asthma with an area under the receiver operator characteristic curve of 0.85 , a sensitivity of 0.79 , and a specificity of $0.84^{[17,19]}$. The Aeonose is an electronic nose device having micro hotplate metal-oxide sensors and demonstrated a lower accuracy and sensitivity compared to the Cyranose 320 when discriminating asthma from healthy controls, whereby, the Aeonose performance results indicate an area under the receiver operator characteristic curve curve of 0.79 , a sensitivity of 0.74 , and a specificity of $0.91^{[17]}$.

Although this section presents and discusses performance results of electronic nose devices configured for use in diagnostic breath analysis applications that have shown moderate to good accuracy in providing a rapid detection of the breath pattern of biomarkers of respiratory disease, electronic noses may also be usefully extended to diagnostic applications of other inflammatory and infectious diseases ${ }^{[15]}$. Due to their portability, ability to rapidly provide point-of-care diagnostic test results without a laboratory technician and their low cost elevate the electronic nose to an attractive diagnostic tool in breath analysis. In regards to low cost, for example, the electronic nose electrical components of, an electronic chemical gas sensor, a microcontroller, and wiring may cost less than approximately $\$ 15.00$ in parts.

\section{ELECTRONIC NOSE SIGNAL PROCESSING CORRECTION}

As a result of metal oxide semiconductor (MOS) based gas sensor commercial availability, low cost, and favorable sensor properties, including a high selectivity to target gases and a short recovery time, the MQ-2 and MQ-135 gas sensors are commonly used gas sensor component for electronic nose technologies for biomedical applications. However, their suitability has yet been determined for use in electronic nose systems directed at real-time monitoring for breath analysis application ${ }^{[20,21]}$. The problem with the use of MOS-based sensors in electronic nose technology is the system experiencing instability due to the output response of the sensor not being under stable control as a result of gradual drifts in the output response of 
the MQ-2 and MQ-135 sensors, resulting in the inability to successfully reproduce output responses using these sensors. For example, a COVID-19 breath simulation study utilized regression analysis to determine the linear model data between Time (s) and the Output Response (V) of an MQ-2 and MQ-135 gas sensor for an electronic nose prototype ${ }^{[22]}$.

The study results determined the correlation values of the output response peaks and the concentration gradient of the sample solution have a fitting formula with a measured relativity $\left(R^{2}\right)>0.9073$ for the MQ-2 gas sensor and a $\mathrm{R}^{2}>0.3963$ for the MQ- 135 gas sensor ${ }^{[22]}$. It would be more desirable to have a gas sensor having an increased reproducibility of more controlled output responses so that acquired gas sensor data obtained from a laboratory setting may be validated and more efficiently applied toward breath analysis applications ${ }^{[23]}$. This baseline instability directly pertains to the baseline output response of the MQ-2 and MQ-135 sensors not being accurately obtained due to factors such as drifts, environmental influences, and sampling means ${ }^{[24]}$. In particular, as it relates to drifts, as a slight variation is observed in MOS-based gas sensor output responses of electronic nose devices having the sensors exposed to an unchanged target gas sample with an unchanged concentration value having the surrounding environment at an ambient status.

Drift in a sensor is a natural phenomenon in which a low frequency change occurs. Drift changes occurring between sample intervals has been investigated to be attributed to many factors including, the degradation of the heating element comprising a nickel-chromium coil surrounded by a layer of aluminum oxide-based ceramic of the sensor over time or damage to the sensing element, comprising a layer of tin dioxide overlaying the heating element, from chemicals that are capable of compromising the integrity of the sensor components ${ }^{[25,26]}$. Further, unstable drift changes of MOS-based gas sensors may result from changes in the environment of the sample chamber such as, temperature, humidity, flow rate of the sample, and concentration of target gas ${ }^{[27]}$. In an example, drift variation is magnified in breath analysis applications when the sensors of an electronic nose device are exposed to a diversity of target gasses within an exhaled breath sample, at varying concentrations, and a changing flow rate corresponding to the inconsistent force of the exhaled breath emitted from an individual's lungs.

In addition, undesirable drift variation exists in MOS-based gas sensors due in part to the sampling mechanism in which the heated tin dioxide layer of the gas sensor is configured for oxygen adsorption on its surface. This oxygen adsorption decreases the flow of electric current flowing through the coil of the MOS gas sensor until the surfaced density of adsorbed oxygen lowers during sensor exposure with a target gas. As a result of target gas exposure to a MOS gas sensor, a resistance change having a value measured using a voltage divider network occurs and is associated with the detection of a specific gas. Thus, in the presence of reducing gases, more electrons are capable of flowing across the coil. The sampling mechanism comprising the electron flow across a coil of a MOS gas sensors requires lengthy cycling time when a saturated sensor refreshes back to baseline drift. This response and recovery time may hinder the accuracy of results and not be suitable for breath analysis applications unless corrected using signal processing ${ }^{[24]}$. Although an efficient cleaning process is a solution to minimizing drifts after each gas exposure phase, the cleaning process requires a length of time that is not suitable for an electronic nose in clinical applications for breath analysis. Thus, there is a need for a signal processing method to minimize the effects of drift on MOS-based gas sensor output responses so that the sensor response drifts are reduced to provide quicker and continuous measurements. As a result, fast measurements as well as maintaining the accuracy of the results are expected from applying the signal processing cleaning step ${ }^{[28]}$.

Current signal correction methods, such as univariate and multivariate are configured for increasing the quality of the sensor output response. In an example, univariate methods are capable of applying the 
correction to one variable, such as the gas sensor during baseline manipulation ${ }^{[29]}$. In particular, the output response of the sensor is altered by the initial measurement of the output response. This alteration is commonly referred to as a transformation and is configured for the correction, such as differential, relative, or fractional, of the sensor's signal baseline. Some studies have indicated the use of baseline manipulation as a means for preliminary processing of the output response of the gas sensor ${ }^{[30]}$. Other studies have utilized various filtering techniques such as Moving Median Filter, Fourier Bandpass Filter, and Discrete Wavelet Transform in an attempt to eliminate adverse drift effects from acquired gas data ${ }^{[30,31]}$. Due to the capabilities of the discrete wavelet transform, a signal may be examined at differing variables such as frequency band levels and resolutions.

Studies have indicated a correlation between output responses of gas sensors and drift effects that have influenced the direction of electronic nose technologies towards utilizing multivariate signal correction methods to apply the correction to many variables, such as a plurality of gas sensors, resulting in an increased amount of data being detected for modeling of nonlinear drift effects ${ }^{[32]}$. In an example, selforganizing maps are a type of adaptive neural network that have been incorporated within the signal processing of some electronic nose sensors ${ }^{[33]}$. Although the use of this adaptive neural network is capable of accomplishing accurate results for the application of gas analysis, the quantification of acquired gas sensor output responses have been identified as a major limitation ${ }^{[3,35]}$. Taking into consideration of the literature review, it can be discerned that drift is associated with the output response of a gas sensor even during exposure of to a target gas having consistent variables such as, temperature, humidity, and time. It would be more desirable to minimize the variance of drift. Further, some studies have investigated and favored the use of orthogonal signal correction (OSC) algorithms to eliminate the variance that is absent any correlation to the variable for approximate calculation ${ }^{[36]}$.

In another example of multivariate linear correction being applied in other studies, the use of Partial Least Square (PLS) and Principal Component Analysis (PCA) has contributed to the decrease in variance of the gas sensor drift as it flows in a single pathway ${ }^{[27,35,37]}$. Although a high accuracy is achieved with PLS and PLS with OSC, when measuring the error of the model in predicting quantitative data, it is more desirable in some applications to have a lower Root Mean Square Error (RMSE) value because the average distance from the predicted values of the model and the dataset values are in closer proximity. A comparison of the stability and accuracy of the regression model of acquired data from an electronic nose prototype with the application of PLS and PLS with OSC resulted in an increased accuracy with both methods, however, the PLS with OSC method required less components than with PLS alone to achieve the same performance ${ }^{[38]}$. In particular, the signal correction method comprising both PLS with OSC for corrected data achieved a RMSE value of approximately $0.12 \%$ using 1 component compared to PLS alone achieving a RMSE value of approximately $0.15 \%$ using 5 components. In a study, OSC was used for the correction of near-infrared spectra (NIR) and many publications described machine learning algorithms to improve the processing of the OSC method to eliminate the variance not correlated to the variable to estimate ${ }^{[38,39]}$.

The signal processing approach to quantification of acquired gas sensor output response data includes applying baseline manipulation with OSC in an attempt to eliminate and/or minimize the drift effects of the gas sensors responding to varying concentrations of an exhaled breath sample. Next, a regression model to determine the approximate concentration of gas detected in ppm is realized after the corrected datasheet undergoes PLS regression. As the gas sensors are exposed to an exhaled breath sample, PLS regression is a good candidate to be applied to model the output responses of the gas sensors. Validation of acquired gas data results are critical for increasing the reproducibility of the sensor output responses for enhancing the regression model stability while maintaining an accuracy suitable for breath analysis applications. It is 
envisioned that this drift compensation method presented will enhance electronic nose design capabilities.

\section{REPORTING DIAGNOSTIC ACCURACY}

The reproducibility, completeness, and transparent reporting of research may be facilitated by the recommendations set forth by the Standards for Reporting Diagnostic Accuracy (STARD) statement ${ }^{[40]}$. Some of the STARD recommendations are directed to the study design, participants, test methods, analysis, test results. For example, a study design method recommendation includes, but is not limited to, identification as a study of diagnostic accuracy such as sensitivity, specificity, predictive values, or AUC ${ }^{[40]}$. As it pertains to the diagnostic testing of individuals for SARS-CoV-2, an electronic nose device having gas sensors with a high selectivity to target gasses would be more desirable. Further, the Transparent Reporting of a multivariable prediction model for Individual Prognosis or Diagnosis (TRIPOD) statement sets forth reporting guidelines for the development and validation of prediction models in diagnostic applications ${ }^{[41]}$. Some of the TRIPOD recommendations are directed to the title, abstract, background and objective, source of data, participants, outcome, predictors, sample size, missing data, statistical analysis methods, risk groups, and development $v$ s. validation. For example, a statistical analysis method recommendation includes, but is not limited to, specify the type of model, all model-building procedures, and methods for internal validation ${ }^{[41]}$. As it pertains to the diagnostic testing of individuals for SARS-CoV-2, an electronic nose device incorporated with OSC, PLS, and PCA to correct drift variations is most desirable.

\section{DISCUSSION OF NEUROLOGICAL MANIFESTATIONS OF COVID-19}

\section{Brain breath biochemistry in neurological complications of COVID-19}

Currently, not many investigations have actively explored the infection mechanism of SARS-CoV-2 and its ability to infiltrate the nervous system. Understanding the biological communication network of the olfactory nerve is essential in expanding the knowledge in regards to SARS-CoV-2 transmission between individuals and metabolic byproducts of infection. It would be particularly useful in the field of diagnostic electronic nose technologies to identify quantitative biomarkers of immune response to include in the breath pattern signature of COVID-19. As a result, electronic nose devices may be fitted with a gas sensor array configured for the detection of disease specific target gasses, SARS-CoV-2 and/or viral components such as the amino acid receptor binding domains (RBDs) of Spike 1 glycoprotein of SARS-CoV-2, and/or a plurality of inflammatory biomarkers emitted from the breath of an infected individual. Infection mechanism of SARS-CoV-2.

Studies have indicated that SARS-CoV-2 has four main structural proteins including, spike (S) glycoproteins, a membrane $(\mathrm{M})$, an envelope $(\mathrm{E})$, and a nucleocapsid $(\mathrm{N})^{[42-45]}$. These $\mathrm{S}$ glycoproteins are embedded on the surface of the outer E portion of SARS-CoV-2 and have a 1273 amino-acid-long structure configured to connect with the human cell membrane protein ACE2 during infection ${ }^{[44,46,47]}$. Studies have shown ACE2 to be located at various host sites such as at the nasal epithelial cells, the heart, the esophagus, the kidneys, the bladder, and the ileum ${ }^{[48]}$. The $S$ glycoprotein is divided into subunit 1 (S1) and subunit 2 $(\mathrm{S} 2)^{[49]}$. S1 is primarily in communication with ACE2 during infection and has beta strands and comprises an N-terminal domain, a first C-terminal domain, a second C-terminal domain, and a third C-terminal domain ${ }^{[50]}$. SARS-CoV-2 binds to the host during infection, studies have shown evidence that some viral proteins contribute to the suppression of cytokines, interferon (IFN)- $\alpha$ and $\beta$ of macrophages during immune respons $\mathrm{e}^{[51]}$. This immune response delay corresponds to the peak of viral load detected in the upper respiratory tract of SARS-CoV-2 infected patients at day 10 of infection ${ }^{[4,5,1]}$. This suppression of IFN- $\alpha$ and $\beta$ may contribute to this infection load peak. 
Although other pathogens of disease may share a similar infection mechanism of SARS-CoV-2, it is an important aspect of this research for the combination of more broadly known neurological inflammatory biomarkers, such as NO, from a plurality of pathogens of disease to be incorporated with the known breath pattern signature of COVID-19. The VOCs of COVID-19 currently consist of specific increases in acetone, isoprene, heptanal, propanol, propanal, butanone, methylpent-2-enal, 2,4-octadiene, 1-chloroheptane, nonanal, octanal, and a decrease in methanol ${ }^{[1,13,14]}$. In regards to diagnostic electronic nose detection of SARS-CoV-2 infection and COVID-19 disease biomarkers, a breath pattern signature having a wider scope including neurological inflammatory biomarkers is a strategy described in this paper to enhance the accuracy of differentiating COVID-19 infection from other pathogens of disease.

\section{Olfactory nerve}

The cribriform plate separates the nasal cavity from the brain ${ }^{[52]}$. The goblet cells, secretory cells, and ciliated cells of the nasal epithelium lines the nasal cavity and paranasal sinuses ${ }^{[53]}$. Olfactory receptor neurons of the olfactory neural cells have a dendritic end portion extending through the olfactory epithelium of the nasal cavity located opposite an end traversing through approximately 15-20 foramina or openings of the cribriform plate and are connected to the olfactory bulb $b^{[7,52,54]}$. The olfactory bulb is positioned within the frontal lobe of the brain and the olfactory tract traverses through the medial temporal lobe of the brain ${ }^{[55]}$. Next, the olfactory tracts divide at the olfactory trigone into three channels. One of the channels is referred to as the lateral olfactory stria which continues laterally, traversing the horizontal Sylvian cistern and ending at the medial temporal lobe approximately at the uncus ${ }^{[56]}$. It has been shown that an infection route of SARS-CoV-2 is through the nasal cavity in communication with the medial temporal lobe of the brain by the olfactory bulb ${ }^{[57]}$.

Studies have confirmed that viral particles up to approximately $100 \mathrm{~nm}$ can enter the CNS through the olfactory epithelium ${ }^{[7]}$. Interestingly, clinical investigations of coronavirus-specific viral particles were observed to range in size from $60-140 \mathrm{~nm}$ in size ${ }^{[58]}$. Thus, SARS-CoV-2 is more than capable of infiltrating the CNS through olfactory receptor neurons and traversing the cribriform plate. Studies have indicated infection of the olfactory bulb epithelium as being a contributor in neurological symptoms in that SARSCoV-2 damages ACE2 and then infiltrates into the blood-brain barrier ${ }^{[59]}$. The unique communication of SARS-CoV-2 with the olfactory receptor neurons of the olfactory epithelium may serve the basis of diagnostic sensing devices and/or canine sensing methods configured for pathogen detection and/or pathogen classification of the nervous system. In younger children, the cribriform plate is undergoing development and may have substantially larger foramina which could result in an increased susceptibility to viral infiltration especially since SARS-CoV-2 is attracted to the ACE2 receptors within the nasal cavity. Studies have indicated that viral transmission along the olfactory nerve is associated with histological lesions ${ }^{[00]}$. These histological lesions in CNS disease have been known to induce an inflammatory response including, but not limited to, NO mediated demyelination and could trigger COVID-19 induced pediatric inflammatory multisystem syndrome ${ }^{[60]}$. Therefore, $\mathrm{NO}$ is a potential biomarker candidates for detecting COVID-19 using an electronic nose device.

\section{Nitric oxide expression in COVID-19 induced PMIS}

A recently observed symptom of COVID-19 is anosmia or loss of smell. Studies have shown an increased ACE2 expression in SARS-CoV-2 infection at the location of nasal epithelial cells ${ }^{[1,53]}$. Because the S1 proteins of SARS-CoV-2 bind to ACE2 for infection to occur, a strong correlation exists between peripheral nerve injury including virus induced olfactory loss and/or injury stemming from viral communication at the olfactory epithelium, resulting in the anosmia symptom of COVID-19. Studies have shown that demyelinating diseases have an increased inflammatory response compared to non-neurological diseases. Hence, this atrophy of the olfactory bulb and olfactory tract might be correlated with demyelination, which 
could trigger an inflammatory response with potentially fatal outcomes in pediatric patients. This inflammatory response induced from COVID-19 can be detected through VOC brain breath analysis with an electronic nose device.

Although, the majority of anosmia observations reported in COVID-19 diagnosed cases appear to be caused from mucosal congestion, peripheral nerve injury could explain anosmia in asymptomatic COVID-19 patients not having mucosal congestion ${ }^{[61]}$. In particular, as a result of the inflammatory response of SARS-CoV-2 connecting with ACE-2 at the olfactory epithelium, macrophages and microglia generate cellular waste such as, inflammatory cytokines and NO. These byproducts contribute to demyelination and axonal $\operatorname{loss}^{[62]}$. NO has been shown to damage mitochondria of axons ${ }^{[63]}$, resulting in a reduction of the enzyme $\mathrm{Na}^{+} / \mathrm{K}^{+}$ATPase, a decrease in ATP supply, and an increase in the amount of cellular Na ATP production is necessary for nervous tissue to maintain stable resting potentials for propagation of electrical signals along the length of nerve fibers. When ATP undergoes hydrolysis and is converted into adenosine diphosphate (ADP) and phosphate, the $\mathrm{Na}^{+} / \mathrm{K}^{+}$pumps become activated again to, thereby, reversing the influx of $\mathrm{Na}^{+}$within the cell ${ }^{[64]}$. There may be a correlation between anosmia as a symptom of COVID-19 with the NO mediated injury to the olfactory receptor neurons.

Although the epithelium of the paranasal sinuses produces NO even without viral infection, macrophages generate excessive $\mathrm{NO}$ as a result of proinflammatory cytokine activation during viral infection defense $\mathrm{e}^{[65,66]}$. When NO and oxygen free radicals, such as the hydroxyl radical $(\mathrm{OH})$ and superoxide $\left(\mathrm{O}_{2}\right)$ are in communication with each other from some viral lung infections, toxic byproducts such as peroxynitrite (ONOO-) and $\mathrm{NOx}\left(\mathrm{NO}_{2}\right.$ and $\left.\mathrm{N}_{2} \mathrm{O}_{3}\right)$ are seen to contribute to the mechanism of viral pneumonia resulting in tissue injuries to the lungs ${ }^{[6,667]}$. Further, scientific evidence has indicated measurable amounts of NO in nasally exhaled air ${ }^{[68]}$. Thus, excessive levels of $\mathrm{NO}$ may be a biomarker of inflammation especially when a biosensor collects breath samples from nasally exhaled air as opposed to orally exhaled air.

\section{CONCLUSION}

Electronic nose technology configured to detect exhaled VOC biomarkers from a breath sample have been observed as a successful approach to noninvasive diagnosis demonstrated in clinical trials for breast cancer, colorectal cancer, lung cancer, liver disease, infectious disease such as influenza and inflammatory lung disease ${ }^{[69]}$. Further, electronic nose technologies are currently used in monitoring air quality, spoiled meats, and ripened fruit; however, its impact on emerging pandemics, such as the respiratory illness pandemic in the form of COVID-19, is still under investigation. Within the coronavirus family, seven viruses are currently known to infect humans, including, NL63 and 229E from the alpha genus and OC43, HKU1, SARS-CoV, MERS-CoV, and SARS-CoV-2 from the beta genus ${ }^{[70]}$. Of these seven viruses, studies have indicated infected SARS-CoV-2 individuals have significant exhaled breath concentrations of ethanal, octanal, acetone, butanone, methylpent-2-enal, 2,4-octadiene, 1-chloroheptane, nonanal, and a decrease in methanol are capable of distinguishing between non-SARS-CoV-2 infected individuals and SARS-CoV-2 infected individuals ${ }^{[1,13,20]}$.

The research results provide for the advancement of the configuration of electronic nose technologies for the application of diagnostic breath analysis of not only SARS-CoV-2, but the remaining six viruses of the coronavirus family, human respiratory viral, and nonviral diseases. For example, studies have probed the role of conducting polymer type electronic nose devices having an array comprising of 32 gas sensors. Although the low power consumption of conducting polymer electronic noses have shown a good sensitivity and reproducibility at room temperatures, their sensitivity to moisture, shortened sensor life, and susceptibility to damage from polar analytes makes MOS-based electronic nose device a more attractive 
alternative for effective detection of disease specific chemical biomarkers for diagnostic applications ${ }^{[7]}$. In summary, breath analysis studies investigating the exhaled breath VOCs of individuals infected with other pathogens of disease may provide clues to specific metabolic byproducts of infection within the coronavirus family. Although it has been shown that SARS-CoV-2 may enter the nasal cavity of a host and traverse the olfactory nerve to generate biomarkers of inflammation within the brain, studies have determined that the circulatory system absorbs these biomarkers which are then ultimately emitted from the lungs through an exhaled breath ${ }^{[71]}$. As our understanding of infectious diseases, such as COVID-19 improves, we expect diagnostic electronic nose devices to include a diversity of MOS-based gas sensors and electrochemical sensors that can more accurately detect the exhaled breath biomarkers of disease as well as changes associated with the pathogenesis of the disease. For diagnostic applications, electronic nose devices are configured for detecting the unique breath pattern signature of a specific disease from a complex mixture of VOCs with the use of a plurality of gas sensors having high sensitivity to targeted biomarkers, thus, improving the disease specificity of the electronic nose device ${ }^{[72]}$.

The highly transmissible nature of SARS-CoV-2 infection of COVID-19 disease has resulted in the need for a rapid COVID-19 breath test, that may serve to diagnose both children and adults around the world. Our research group has investigated the SARS-CoV-2 infection mechanisms and its inflammatory response by the central nervous system by analyzing the symptoms of COVID-19 and similar diseases while partnering with breath specialists that have collected, measured, and validated VOCs from breath samples of COVID-19 positive individuals. Substantial evidence suggests VOCs associated with the inflammatory response of a COVID-19 infection may include, but are not limited to, NO, alcohol, and acetone. These VOCs are capable of being detected when they are exhaled through the breath of an individual. A rapid COVID-19 breath test with an electronic nose gas sensor array configured for noninvasive and rapid detection of the breath pattern signature of COVID-19 may contribute to a substantial reduction of the transmission rate of the global health threat of infection and result in a reduction of COVID-19 related deaths.

\section{DECLARATIONS}

\section{Acknowledgment}

Since the beginning of the COVID-19 outbreak, it has caused an incredible amount of damage to humans as well as economic repercussions. We thank The University of South Florida COVID-19 Rapid Response Research Grants program and the efforts made by the University of South Florida researchers for their contributions to high impact research and public service during this global pandemic.

\section{Authors' contributions}

Wrote the manuscript: Miller TC, Morgera SD, Saddow SE, Takshi A, Mullarkey M, Palm M

Provided critical revision and final approval of the article: Miller TC, Morgera SD

\section{Availability of data and materials}

Not applicable.

\section{Financial support and sponsorship}

This work is supported by a University of South Florida Pandemic Response Research Network funding for COVID-19 to Dr. Salvatore D. Morgera.

\section{Conflicts of interest}

All authors declared that there are no conflicts of interest. 


\section{Ethical approval and consent to participate}

Not applicable.

\section{Consent for publication}

Not applicable.

\section{Copyright}

(c) The Author(s) 2021.

\section{REFERENCES}

1. Ruszkiewicz DM, Sanders D, O'Brien R, et al. Diagnosis of COVID-19 by analysis of breath with gas chromatography-ion mobility spectrometry - a feasibility study. EClinicalMedicine 2020;29:100609. DOI PubMed PMC

2. Henderson B, Ruszkiewicz DM, Wilkinson M, et al. A benchmarking protocol for breath analysis: the peppermint experiment. $J$ Breath Res 2020;14:046008. DOI PubMed

3. Boesveldt S, Postma EM, Boak D, et al. Anosmia-a clinical review. Chem Senses 2017;42:513-23. DOI PubMed PMC

4. Marinosci A, Landis BN, Calmy A. Possible link between anosmia and COVID-19: sniffing out the truth. Eur Arch Otorhinolaryngol 2020;277:2149-50. DOI PubMed PMC

5. Palao M, Fernández-Díaz E, Gracia-Gil J, Romero-Sánchez CM, Díaz-Maroto I, Segura T. Multiple sclerosis following SARS-CoV-2 infection. Mult Scler Relat Disord 2020;45:102377. DOI PubMed PMC

6. Wang L, Shen Y, Li M, et al. Clinical manifestations and evidence of neurological involvement in 2019 novel coronavirus SARSCoV-2: a systematic review and meta-analysis. J Neurol 2020;267:2777-89. DOI PubMed PMC

7. Riel D, Verdijk R, Kuiken T. The olfactory nerve: a shortcut for influenza and other viral diseases into the central nervous system. $J$ Pathol 2015;235:277-87. DOI PubMed

8. Wang P. Combination of serological total antibody and RT-PCR test for detection of SARS-COV-2 infections. J Virol Methods 2020;283:113919. DOI PubMed PMC

9. Rannan-Eliya RP, Wijemunige N, Gunawardana JRNA, et al. Increased intensity of PCR testing reduced COVID-19 transmission within countries during the first pandemic wave. Health Aff (Millwood) 2021;40:70-81. DOI PubMed

10. Garg A, Ghoshal U, Patel SS, et al. Evaluation of seven commercial RT-PCR kits for COVID-19 testing in pooled clinical specimens. J Med Virol 2021;93:2281-6. DOI PubMed PMC

11. Kanji JN, Zelyas N, MacDonald C, et al. False negative rate of COVID-19 PCR testing: a discordant testing analysis. Virol J 2021;18:13. DOI PubMed PMC

12. Grover A, Lall B. A novel method for removing baseline drifts in multivariate chemical sensor. IEEE Trans Instrum Meas 2020;69:7306-16. DOI

13. Grassin-Delyle S, Roquencourt C, Moine P, et al; Garches COVID-19 Collaborative Group RECORDS Collaborators and Exhalomics ${ }^{\circledR}$ Collaborators. Metabolomics of exhaled breath in critically ill COVID-19 patients: a pilot study. EBioMedicine 2021;63:103154. DOI PubMed PMC

14. Giovannini G, Haick H, Garoli D. Detecting COVID-19 from Breath: a game changer for a big challenge. ACS Sens 2021;6:1408-17. DOI PubMed PMC

15. Farraia MV, Cavaleiro Rufo J, Paciência I, Mendes F, Delgado L, Moreira A. The electronic nose technology in clinical diagnosis: a systematic review. Porto Biomed J 2019;4:e42. DOI PubMed PMC

16. Dragonieri S, Pennazza G, Carratu P, Resta O. Electronic nose technology in respiratory diseases. Lung 2017;195:157-65. DOI PubMed

17. Licht JC, Grasemann H. Potential of the electronic nose for the detection of respiratory diseases with and without Infection. Int $J$ Mol Sci 2020;21:9416. DOI PubMed PMC

18. Dragonieri S, Schot R, Mertens BJ, et al. An electronic nose in the discrimination of patients with asthma and controls. $J$ Allergy Clin Immunol 2007;120:856-62. DOI PubMed

19. Tenero L, Sandri M, Piazza M, Paiola G, Zaffanello M, Piacentini G. Electronic nose in discrimination of children with uncontrolled asthma. J Breath Res 2020;14:046003. DOI PubMed

20. Wilson AD, Baietto M. Advances in electronic-nose technologies developed for biomedical applications. Sensors (Basel) 2011;11:1105-76. DOI PubMed PMC

21. Kalidoss R, Umapathy S. An overview on the exponential growth of non-invasive diagnosis of diabetes mellitus from exhaled breath by nanostructured metal oxide Chemi-resistive gas sensors and $\mu$-preconcentrator. Biomed Microdevices 2019;22:2. DOI PubMed

22. Miller TC, Morgera SD, Saddow SE, Takshi A, Palm M. Electronic nose with detection method for alcohol, acetone, and carbon monoxide in coronavirus disease 2019 breath simulation model. IEEE Sensors J 2021:1-9. DOI

23. Marco S, Gutierrez-galvez A. Signal and data processing for machine olfaction and chemical sensing: a review. IEEE Sensors $J$ 2012;12:3189-214. DOI

24. Monroy JG, González-Jiménez J, Blanco JL. Overcoming the slow recovery of MOX gas sensors through a system modeling approach. Sensors (Basel) 2012;12:13664-80. DOI PubMed PMC

25. Korotcenkov G, Cho B. Instability of metal oxide-based conductometric gas sensors and approaches to stability improvement (short 
survey). Sens Actuators B Chem 2011;156:527-38. DOI

26. Sharma RK, Chan PC, Tang Z, Yan G, Hsing I, Sin JK. Investigation of stability and reliability of tin oxide thin-film for integrated micro-machined gas sensor devices. Sens Actuators B Chem 2001;81:9-16. DOI

27. Natale C, Martinelli E, D'amico A. Counteraction of environmental disturbances of electronic nose data by independent component analysis. Sens Actuators B Chem 2002;82:158-65. DOI

28. Wold S, Antti H, Lindgren F, Öhman J. Orthogonal signal correction of near-infrared spectra. Chemom Intell Lab Syst 1998;44:17585. DOI

29. Hines E, Llobet E, Gardner J. Electronic noses: a review of signal processing techniques. IEE Proc, Circuits Devices Syst 1999;146:297. DOI

30. Gardner JW, Bartlett PN. Electronic noses. Principles and applications. Meas Sci Technol 2000;11:1087.

31. Llobet E, Brezmes J, Ionescu R, et al. Wavelet transform and fuzzy ARTMAP-based pattern recognition for fast gas identification using a micro-hotplate gas sensor. Sens Actuators B Chem 2002;83:238-44. DOI

32. Zuppa M, Distante C, Persaud KC, Siciliano P. Recovery of drifting sensor responses by means of DWT analysis. Sens Actuators $B$ Chem 2007;120:411-6. DOI

33. Ziyatdinov A, Marco S, Chaudry A, Persaud K, Caminal P, Perera A. Drift compensation of gas sensor array data by common principal component analysis. Sens Actuators B Chem 2010;146:460-5. DOI

34. Marco S, Ortega A, Pardo A, Samitier J. Gas identification with tin oxide sensor array and self-organizing maps: adaptive correction of sensor drifts. IEEE Trans Instrum Meas 1998;47:316-21. DOI

35. Fenske JD, Paulson SE. Human breath emissions of VOCs. J Air Waste Manag Assoc 1999;49:594-8. DOI PubMed

36. Artursson T, Eklöv T, Lundström I, Mårtensson P, Sjöström M, Holmberg M. Drift correction for gas sensors using multivariate methods. J Chemom 2000;14:711-23. DOI

37. Ding H, Liu JH, Shen ZR. Drift reduction of gas sensor by wavelet and principal component analysis. Sens Actuators $B$ Chem 2003;96:354-63. DOI

38. Laref R, Ahmadou D, Losson E, Siadat M. Orthogonal signal correction to improve stability regression model in gas sensor systems. $J$ Sens 2017;2017:1-8. DOI

39. Ahmadou D, Laref R, Losson E, Siadat M. Reduction of drift impact in gas sensor response to improve quantitative odor analysis. In: Proceedings of the IEEE International Conference on Industrial Technology; 2017 Mar 22-25; Toronto, ON, Canada. 2017. p. 928-33. DOI

40. Bossuyt PM, Reitsma JB, Bruns DE, et al; STARD Group. STARD 2015: an updated list of essential items for reporting diagnostic accuracy studies. BMJ 2015;351:h5527. DOI PubMed PMC

41. Moons KG, Altman DG, Reitsma JB, et al. Transparent reporting of a multivariable prediction model for individual prognosis or diagnosis (TRIPOD): explanation and elaboration. Ann Intern Med 2015;162:W1-73. DOI PubMed

42. Seo G, Lee G, Kim MJ, et al. Rapid detection of COVID-19 causative virus (SARS-CoV-2) in human nasopharyngeal swab specimens using field-effect transistor-based biosensor. ACS Nano 2020;14:5135-42. DOI PubMed PMC

43. Gao L, Ding YS, Dai H, Huang ZD, Shao SH. A novel fingerprint map of SARS-CoV with visualization analysis. Third International Conference on Image and Graphics (ICIG'04), 2004, p. 226-9. DOI

44. Wang Q, Zhang Y, Wu L, et al. Structural and functional basis of SARS-CoV-2 entry by using human ACE2. Cell 2020;181:894904.e9. DOI PubMed PMC

45. Hoffmann M, Hofmann-winkler H, Pöhlmann S. Priming time: how cellular proteases arm coronavirus spike proteins. In: Böttcherfriebertshäuser E, Garten W, Klenk HD, editors. Activation of Viruses by Host Proteases. Cham: Springer International Publishing; 2018. p. 71-98. DOI

46. Ortega JT, Serrano ML, Pujol FH, Rangel HR. Role of changes in SARS-CoV-2 spike protein in the interaction with the human ACE2 receptor: an in silico analysis. EXCLI J 2020;19:410-7. DOI PubMed PMC

47. Walls AC, Park YJ, Tortorici MA, Wall A, McGuire AT, Veesler D. Structure, function, and antigenicity of the SARS-CoV-2 spike glycoprotein. Cell 2020;181:281-92.e6. DOI PubMed PMC

48. Dong D, Tang Z, Wang S, et al. The role of imaging in the detection and management of COVID-19: a review. IEEE Rev Biomed Eng 2021;14:16-29. DOI PubMed

49. Cascella M, Rajnik M, Cuomo A, Dulebohn SC, Di Napoli R. Features, evaluation and treatment coronavirus (COVID-19). In: StatPearls [Internet]. Treasure Island (FL): StatPearls Publishing; 2021. PubMed

50. Gui M, Song W, Zhou H, et al. Cryo-electron microscopy structures of the SARS-CoV spike glycoprotein reveal a prerequisite conformational state for receptor binding. Cell Res 2017;27:119-29. DOI PubMed PMC

51. Cheung CY, Poon LL, Ng IH, et al. Cytokine responses in severe acute respiratory syndrome coronavirus-infected macrophages in vitro: possible relevance to pathogenesis. J Virol 2005;79:7819-26. DOI PubMed PMC

52. Doty RL, Kamath V. The influences of age on olfaction: a review. Front Psychol 2014;5:20. DOI PubMed PMC

53. Sungnak W, Huang N, Bécavin C, et al; HCA Lung Biological Network. SARS-CoV-2 entry factors are highly expressed in nasal epithelial cells together with innate immune genes. Nat Med 2020;26:681-7. DOI PubMed

54. Wenig BM. Undifferentiated malignant neoplasms of the sinonasal tract. Arch Pathol Lab Med 2009;133:699-712. DOI PubMed

55. Olfactory Pathways and Limbic System. Available from: http://www.neuroanatomy.wisc.edu/coursebook/neuro3(2).pdf. [Last accessed on 27 Jul 2021].

56. Borden NM, Forseen SE, Stefan C. Imaging anatomy of the human brain: a comprehensive atlas including adjacent structures. New York: Springer Medical Publishing. 2016. DOI 
57. Montalvan V, Lee J, Bueso T, De Toledo J, Rivas K. Neurological manifestations of COVID-19 and other coronavirus infections: a systematic review. Clin Neurol Neurosurg 2020;194:105921. DOI PubMed PMC

58. Kim JM, Chung YS, Jo HJ, et al. Identification of coronavirus isolated from a patient in Korea with COVID-19. Osong Public Health Res Perspect 2020;11:3-7. DOI PubMed PMC

59. Ramos FJ, Palomino García A, Jiménez Hernández MD. Neurology during the pandemic. Is COVID-19 changing the organisation of neurology departments? Neurologia (Engl Ed) 2020;35:269-71. DOI PubMed PMC

60. Ryan MA, Zhou H, Buehler MG, et al. Monitoring space shuttle air quality using the Jet Propulsion Laboratory electronic nose. IEEE Sens J 2004;4:337-47. DOI PubMed

61. Galougahi MK, Ghorbani J, Bakhshayeshkaram M, Naeini AS, Haseli S. Olfactory bulb magnetic resonance imaging in SARS-CoV-2induced anosmia: the first report. Acad Radiol 2020;27:892-3. DOI PubMed PMC

62. Caruso D, Pesaresi M, Maschi O, Giatti S, Garcia-Segura LM, Melcangi RC. Effect of short-and long-term gonadectomy on neuroactive steroid levels in the central and peripheral nervous system of male and female rats. J Neuroendocrinol 2010;22:1137-47. DOI PubMed

63. Waxman SG. Axonal conduction and injury in multiple sclerosis: the role of sodium channels. Nat Rev Neurosci 2006;7:932-41. DOI PubMed

64. Miller TC. Brain-computer interface based on magnetic particle imaging for diagnostic and neurological rehabilitation in multiple sclerosis. In: Proceedings of the 8th International Winter Conference on Brain-Computer Interface (BCI), Brain-Computer Interface (BCI); 2020 Feb 26-28; Gangwon, Korea (South). 2020. p. 1-6. DOI

65. Stefano GB, Esch T, Kream RM. Potential immunoregulatory and antiviral/SARS-CoV-2 activities of nitric oxide. Med Sci Monit 2020;26:e925679. DOI PubMed PMC

66. Babizhayev MA, Deyev AI. Management of the virulent influenza virus infection by oral formulation of nonhydrolized carnosine and isopeptide of carnosine attenuating proinflammatory cytokine-induced nitric oxide production. Am J Ther 2012;19:e25-47. DOI PubMed

67. Akaike T, Suga M, Maeda H. Free radicals in viral pathogenesis: molecular mechanisms involving superoxide and NO. Proc Soc Exp Biol Med 1998;217:64-73. DOI PubMed

68. Lundberg JO. Airborne nitric oxide: inflammatory marker and aerocrine messenger in man. Acta Physiol Scand Suppl 1996;633:1-27. PubMed

69. Lourenço C, Turner C. Breath analysis in disease diagnosis: methodological considerations and applications. Metabolites 2014;4:46598. DOI PubMed PMC

70. Abdelrahman Z, Li M, Wang X. Comparative review of SARS-CoV-2, SARS-CoV, MERS-CoV, and influenza a respiratory viruses. Front Immunol 2020;11:552909. DOI PubMed PMC

71. Wilson AD. Advances in electronic-nose technologies for the detection of volatile biomarker metabolites in the human breath. Metabolites 2015;5:140-63. DOI PubMed PMC

72. Wilson AD. Biomarker metabolite signatures pave the way for electronic-nose applications in early clinical disease diagnoses. Curr Metabolom 2017;5:90-101. DOI 